\title{
Seismic response of linear and non-linear asymmetric systems with non-linear fluid viscous dampers
}

\author{
Rakesh K. Goel ${ }^{* \dagger}$ \\ Department of Civil and Environmental Engineering, California Polytechnic State University, \\ San Luis Obispo, CA 93407, U.S.A.
}

\begin{abstract}
SUMMARY
This investigation is concerned with the seismic response of one-story, one-way asymmetric linear and non-linear systems with non-linear fluid viscous dampers. The seismic responses are computed for a suite of 20 ground motions developed for the SAC studies and the median values examined. Reviewed first is the behaviour of single-degree-of-freedom systems to harmonic and earthquake loading. The presented results for harmonic loading are used to explain a few peculiar trends-such as reduction in deformation and increase in damper force of short-period systems with increasing damper non-linearity-for earthquake loading. Subsequently, the seismic responses of linear and non-linear asymmetric-plan systems with non-linear dampers are compared with those having equivalent linear dampers. The presented results are used to investigate the effects of damper non-linearity and its influence on the effects of plan asymmetry. Finally, the design implications of the presented results are discussed. Copyright (C) 2005 John Wiley \& Sons, Ltd.
\end{abstract}

KEY WORDS: asymmetric buildings; inelastic response; passive control; non-linear fluid viscous damper; earthquake response; torsion; supplemental damping

\section{INTRODUCTION}

Several recent studies have shown that supplemental fluid viscous damping effectively reduces the seismic response of asymmetric-plan systems [1-5]. However, these investigations examined the behavior of asymmetric-plan systems with linear fluid viscous dampers. Nonlinear fluid viscous dampers (velocity exponent less than one) have the apparent advantage of limiting the peak damper force at large velocities while still providing sufficient supplemental damping [5-8]; for linear dampers (velocity exponent equal to one), the damper force increases linearly with damper velocity [9-11].

\footnotetext{
*Correspondence to: Rakesh K. Goel, Department of Civil and Environmental Engineering, California Polytechnic State University, San Luis Obispo, CA 93407, U.S.A.

†E-mail: rgoel@calpoly.edu
}

Contract/grant sponsor: Science Foundation; contract/grant number: CMS-9812414 
A recent investigation examined the seismic response of asymmetric systems with non-linear viscous and viscoelastic dampers [12]. It was found that structural response is weakly affected by damper non-linearity, and non-linear dampers achieve essentially the same reduction in response but with much smaller damper force compared to linear dampers; reductions up to $20 \%$ were observed for edge deformations and plan rotations of short-period systems. Furthermore, it was shown that the earthquake response of the asymmetric systems with non-linear dampers can be estimated with sufficient degree of accuracy by analyzing the same asymmetric systems with equivalent linear dampers. A simplified analysis procedure for asymmetric-plan systems with non-linear dampers has also been developed [13].

While the aforementioned investigations have led to improved understanding of the effects of damper non-linearity on the seismic response of asymmetric systems, the following aspects of the problem still need further investigation. First, an earlier investigation by the author [14] indicated that the force in a non-linear damper may be higher than that in a similarly sized linear damper in very-short-period systems. This contradicts the conclusion in many earlier investigations, including Lin and Chopra [12], that the force in a non-linear damper is smaller than in a linear damper. Note that the results presented in Figure 16 of the paper by Lin and Chopra [12] indicated higher force in non-linear dampers for very-short-period systems, but no explanation was provided for such behaviour. This behaviour needs further investigation and explanation.

Second, most previous investigations are limited to systems with lateral load resisting elements responding in the linear elastic range of behaviour. Although it is desirable to limit the seismic performance of buildings to the linear elastic range by using supplemental damping, limited damage is permitted in such buildings during the design earthquake for economical reasons as is apparent from the commentary in the FEMA-356 document [15] which states that supplemental damping 'devices would be expected to be good candidates for projects that have a target Building Performance Level of Life safety or perhaps Immediate Occupancy'. Therefore, there is a need to investigate the effects of damper non-linearity on the seismic response of asymmetric systems responding beyond the elastic range (or non-linear asymmetric systems).

The investigation by Lin and Chopra [12] examined the effects of damper non-linearity on edge deformations and damper forces. For asymmetric-plan systems, however, other important response quantities of interest for design purposes include base shear, base torque, and base torque generated by asymmetric distribution of dampers. Therefore, it is useful to investigate the effects of damper non-linearity on these responses.

The research reported in this paper is aimed at addressing the aforementioned needs as well as independently verifying the findings of earlier investigations. For this purpose, a comprehensive investigation was conducted to examine: (1) the effects of damper non-linearity on the seismic response of linear and non-linear asymmetric systems; and (2) how the effects of plan asymmetry are influenced by the damper non-linearity.

In order to facilitate the selection of damper properties, summarized first is the theoretical background on non-linear fluid viscous dampers followed by the behaviour of single-degreeof-freedom (SDOF) systems with non-linear fluid viscous dampers subjected to harmonic and earthquake loading. The results for SDOF systems, some of which may appear to be duplicative of results presented in earlier publications, e.g. Reference [5], are included here because they explain the trends found later for asymmetric-plan systems. The effects of damper non-linearity on the seismic response of asymmetric-plan systems are investigated next by 
comparing seismic responses - edge deformations, base shear and torque, and total damping force and torque at the base - of one-story, one-way asymmetric systems with non-linear fluid viscous dampers (velocity exponent $=0.7,0.5$, and 0.35 ) and those with linear fluid viscous dampers (velocity exponent $=1$ ). Finally, the influence of damper non-linearity on planasymmetry effects is investigated by comparing the ratio of response of asymmetric- and its corresponding symmetric-plan systems for various values of $(=1,0.7,0.5$, and 0.35$)$; the corresponding symmetric-plan system is defined later in this paper. The seismic responses are computed for a wide range of system parameters and a suite of 20 ground motions developed for the SAC studies [16].

\section{THEORETICAL BACKGROUND: NON-LINEAR DAMPERS}

The force in a non-linear fluid viscous damper is given by

$$
f_{\mathrm{D}}=C \operatorname{sgn}(\dot{u})|\dot{u}|
$$

in which $C$ is the damper coefficient, $\dot{u}$ is the damper velocity, $\operatorname{sgn}(\cdot)$ is the signum function, and is the damper exponent ranging in values from 0.2 to 1 for seismic applications $[6,8,10,17]$. For $=1$, Equation (1) becomes $f_{\mathrm{D}}=C_{1} \dot{u}$ which represents force in a linear damper. Therefore, exponent is representative of the non-linearity of a fluid viscous damper.

For a single-degree-of-freedom (SDOF) system with mass $m$, stiffness $k$, and a non-linear fluid viscous damper defined by Equation (1), the supplemental damping ratio sd is generally defined based on the concept of equivalent linear viscous damping $[6,10,11,17]$ as:

$$
\mathrm{sd}=\frac{E_{\mathrm{D}}}{4 \pi E_{\mathrm{So}}}=\frac{E_{\mathrm{D}}}{2 \pi k u_{\mathrm{o}}^{2}}
$$

where $E_{\mathrm{So}}$ is the elastic energy stored at the maximum system displacement, $u_{0}$, and $E_{\mathrm{D}}$ is the energy dissipated during one cycle of harmonic motion $u=u_{\mathrm{o}} \sin \omega t$ at $\omega=\omega_{\mathrm{n}}\left(\omega_{\mathrm{n}}=\right.$ natural frequency of the SDOF system). For a non-linear damper, $E_{\mathrm{D}}$ is given by $[6,8,10,17]$ :

$$
E_{\mathrm{D}}=\quad C \omega_{\mathrm{n}} u_{\mathrm{o}}^{1+}
$$

where the constant is

$$
=\frac{2^{2+}{ }^{2}(1+\alpha / 2)}{\Gamma(2+)}
$$

and $\Gamma(\cdot)$ is the gamma function; Equation (4) can also be written in an alternative but equivalent form [18]. Utilizing Equation (3) in Equation (2) gives $\mathrm{sd}$ as a function of the peak displacement $u_{\mathrm{o}}$ :

$$
\mathrm{sd}=\frac{C}{2 m \omega_{\mathrm{n}}}\left(\omega_{\mathrm{n}} u_{\mathrm{o}}\right)^{-1}
$$

Therefore, for a given value of supplemental damping ratio, sd, the damper coefficient of a non-linear damper with damper exponent of can be calculated as

$$
C=\frac{2 m \omega_{\mathrm{n} \text { sd }}}{C}\left(\omega_{\mathrm{n}} u_{\mathrm{o}}\right)^{1-}
$$




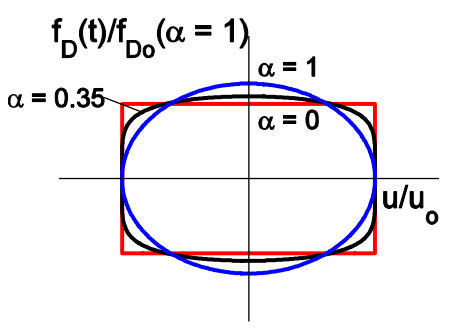

(a)

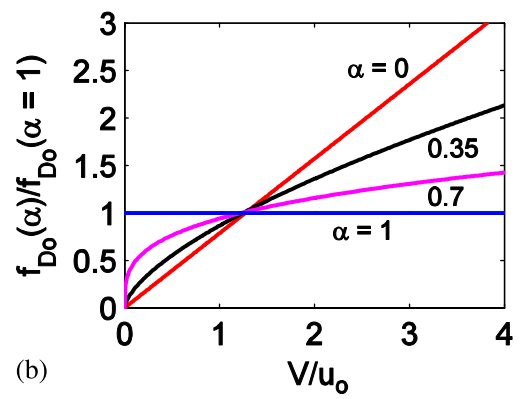

(b)

Figure 1. (a) Hysteresis loops for linear $(=1)$ and non-linear $(=0.35$ and 0$)$ dampers; and (b) ratio of damper force in non-linear and linear damper having the same equivalent damping ratio.

For a linear damper with $=1$, Equation (6) gives $C_{1}=2 m \omega_{\mathrm{n} \text { sd }}$ implying that the damping coefficients of non-linear and linear dampers, both with the same damping ratio, sd, are related as:

$$
C=\frac{\left(\omega_{\mathrm{n}} u_{\mathrm{o}}\right)^{1-}}{C_{1}}
$$

Using equation (7), Equation (1) can be re-written as:

$$
\frac{f_{\mathrm{D}}(t)}{f_{\mathrm{Do}}(=1)}=\frac{1}{\dot{u}_{\mathrm{o}}} \frac{1}{-}\left(\omega_{\mathrm{n}} u_{\mathrm{o}}\right)^{1-} \operatorname{sgn}(\dot{u})|\dot{u}|
$$

and the peak value of the damper force is given as:

$$
\frac{f_{\mathrm{Do}}()}{f_{\mathrm{Do}}(=1)}=\frac{1}{\left(\frac{V}{\dot{u}_{\mathrm{o}}}\right)^{1-}}
$$

in which $V=\omega_{\mathrm{n}} u_{\mathrm{o}}$ is the pseudo-velocity for the SDOF system. The relationship obtained in Equation (9) assumes that the peak velocity of a SDOF system with non-linear damper is identical to that of the corresponding system with linear damper.

Figure 1(a) presents the force-displacement response (or hysteresis loops) of linear $(=1)$ and non-linear $(=0.35$ and 0$)$ fluid viscous dampers with equivalent damping ratio sd when subjected to harmonic motion. The hysteresis loop for the linear damper $(=1)$ is a wellknown elliptical shape whereas that of the non-linear damper with $=0$ (friction damper) is rectangular; the shape for the non-linear damper with $0<\alpha<1$ falls between these two extremes. Because all hysteresis loops enclose the same area ( $\mathrm{sd}$ was defined based on equal energy dissipation or equal area), the peak damper force in the non-linear damper $(\alpha<1)$ is less than that for the linear damper $(=1)$. For systems subjected to harmonic motion, Equation (9) simplifies to $f_{\text {Do }}() / f_{\text {Do }}()=1 / \beta$, which gives $0.785(=\pi / 4)$ for $=0$ and 0.866 for $=0.35$. This indicates that the peak damper force in friction damper is about $22 \%$ and $13 \%$ less in non-linear dampers with $=0$ and 0.35 , respectively, compared to the linear damper.

Figure 1(b) presents the relationship between the damper force and the ratio of the pseudo-velocity and peak velocity, $V / \dot{u}_{\mathrm{o}}$ (Equation (9)). For small values of $V / \dot{u}_{\mathrm{o}}$, the force 

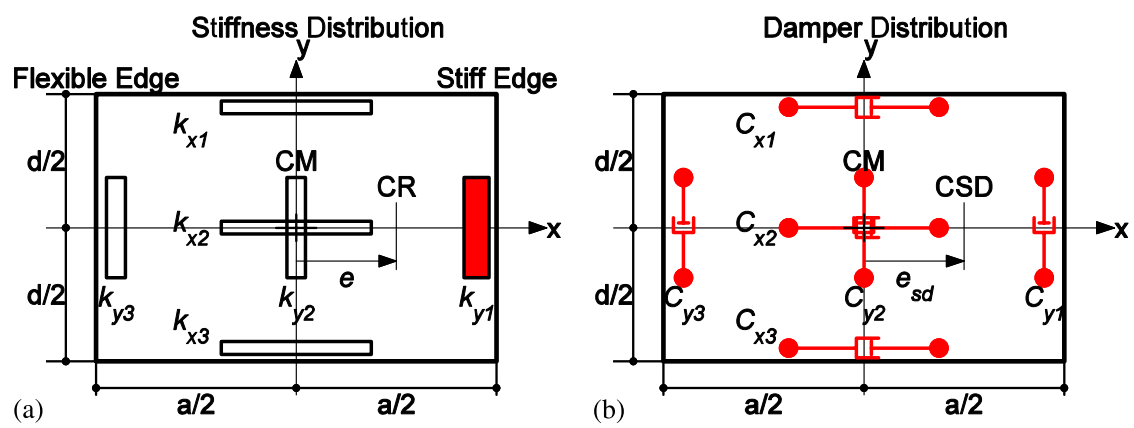

Figure 2. The one-story, one-way asymmetric system considered: (a) locations of lateral force resisting elements; and (b) locations of fluid viscous dampers.

in a non-linear damper is smaller than that in the linear damper. However, the force in a non-linear damper may become larger than that in a linear damper for larger values of $V / \dot{u}_{\mathrm{o}}$. The threshold value of $V / \dot{u}_{\mathrm{o}}$ at which the non-linear damper force becomes larger depends on and is slightly larger than $V / \dot{u}_{\mathrm{o}}=1$; transition occurs at a value of the $V / \dot{u}_{\mathrm{o}}=1 /(1-)$ ( $=1.273$ and 1.248 for $=0$ and 0.35 , respectively).

\section{SYSTEMS, GROUND MOTIONS, AND RESPONSE STATISTICS}

\section{Asymmetric-plan system}

The asymmetric-plan system considered is the idealized one-story building (Figure 2) consisting of a rigid deck supported by structural elements (wall, columns, moment-frames, bracedframes, etc.), and fluid viscous dampers incorporated into the bracing system. The mass properties of the system were assumed to be symmetric about both the $x$-and $y$-axes whereas the stiffness and the damper properties were considered to be symmetric only about the $x$-axis.

The Center of Mass (CM), Center of Rigidity (CR), and Center of Supplemental Damping (CSD) are defined as follows. The CM of the system is the centroid of inertia forces when the system is subjected to a uniform translational acceleration in the direction under consideration. Since the mass was uniformly distributed about both the $x$-and $y$-axes, the CM coincided with the geometric center of the deck. The CR is the point on the deck through which application of a static horizontal force causes no rotation of the deck. The lack of symmetry in the stiffness properties about the $y$-axis was characterized by the stiffness eccentricities, $e$, defined as the distance between the CM and the CR. With both CM and CR defined, the edge that is on the same side of the CM as the CR was denoted as the stiff edge and the other edge was designated as the flexible edge (Figure 1(a)). The CSD is the centroid of damper forces when the system is subjected to a uniform translational velocity in the direction under consideration. The lack of symmetry in the damper properties about the $y$-axis was characterized by the supplemental damping eccentricity, $e_{\text {sd }}$, defined as the distance between the CM and the CSD (Figure 1(b)).

The lateral force resisting system consists of six elements, three each in the $x$ - and $y$-directions (Figure 2(a)). The middle element in each direction is located at the CM and the 
two outermost elements are equidistant from the CM. Since the stiffness eccentricity in the $y$-direction is zero, the three elements in the $x$-direction have equal stiffness, i.e. $k_{x 1}=k_{x 2}=k_{x 3}$. In the $y$-direction, elements 2 and 3 possess equal stiffness, i.e. $k_{y 2}=k_{y 3}$, while the stiffness of element 1 is larger than those of elements 2 and 3; the relative values depend on the stiffness eccentricity in the $x$-direction.

The supplemental damping distribution also consists of six dampers, three each in the $x$ - and $y$-directions (Figure 2(b)). The two outermost dampers in each direction are located at the two edges and the middle damper is located at the CM. The total damping in the $x$ - and $y$-directions are assumed to be equal. Furthermore, the damper distribution in the $x$-direction is assumed to be symmetric, i.e. $C_{x 1}=C_{x 2}=C_{x 3}$. In the $y$-direction, $C_{y 2}=C_{y 3}$, and $C_{y 1}>C_{y 2}$ or $C_{y 3}$. The total value of damper coefficient in a selected direction, defined by the iterative procedure described next, is distributed to various dampers based on the damping eccentricity in the $x$-direction. The procedure to determine the distribution of damper coefficients is available elsewhere [14,19].

The non-linear damper coefficient, $C$, given by Equation (6), depends on the peak displacement of the system, $u_{0}$, for a selected value of the equivalent supplemental damping ratio, sd. Since $u_{\mathrm{o}}$ is not known before the start of the analysis, the following iterative procedure is used to compute the damper coefficient $C$.

1. Assume an initial value of $u_{\mathrm{o}}^{(0)}$ and compute the value of $C^{(0)}$ from Equation (6). An upper-bound estimate of $u_{\mathrm{o}}^{(0)}$ may be obtained from the elastic response spectrum of an SDOF system with linear viscous damping equal to the natural damping ratio of the system plus the supplemental damping ratio.

2. Compute the peak response $u_{\mathrm{o}}^{(1)}$ of an elastic SDOF system with non-linear damper to selected loading from direct solution of the equation of motion.

3. For the $i$-th iteration, set $u_{\mathrm{o}}^{(i)}=\left(u_{\mathrm{o}}^{(i)}+u_{\mathrm{o}}^{(i-1)}\right) / 2$, determine $C^{(i)}$ from Equation (6), and recompute the response from direct solution of the equation of motion of an elastic SDOF system with non-linear damper.

4. Repeat step (3) until two successive values of $u_{\mathrm{o}}$ are sufficiently close. Compute final value of $C$ from Equation (6) for the last value of $u_{\mathrm{o}}$.

The updated estimate of $u_{\mathrm{o}}$ in step (3) of the iterative procedure is based on the average of the previous two estimates. The estimate based on the previous value was not used because it did not lead to convergence for all cases. The first (and adopted) procedure always led to convergence, although at somewhat slower rate compared to the second procedure.

For a selected value of ${ }_{s d}$, the value of $C$ is always computed for an elastic SDOF system because the relationship described by Equation (6) is restricted to systems vibrating at amplitudes within the linearly elastic limit of the overall structure [11]. As a result, the total supplemental damping coefficient, $C$, for the non-linear system is equal to that of the corresponding linear system. However, the effective supplemental damping ratio, which depends on the peak response of the system (Equation (5)), may be slightly different for the two systems.

The aforementioned iterative procedure to determine $C$ ensures that the damping ratio for all earthquake ground motions in a selected ensemble would be the same. An alternative, but simpler approach would be to compute the damping coefficient $C$ for $u_{\mathrm{o}}$ obtained from median linear spectra for the selected ensemble of ground motions constructed for a damping ratio equal to the natural damping ratio of the system plus the supplemental damping ratio. While 
this procedure eliminates the need for iterations, it may lead to slightly different equivalent damping ratios for different earthquakes. This approach was adopted in the research reported in earlier publications $[14,19]$, but it did not lead to conclusions significantly different from those reported in this paper.

For non-linear systems, the total strength of the system is defined by reducing the strength required for the system to remain elastic by a reduction factor $R$, an approach consistent with most seismic design codes and recommendations. The reduction factor is applied to the strength of the elastic system with natural and supplemental damping. The total strength is distributed to the various lateral load-resisting elements in proportion to their stiffness. The stiffness-proportional strength distribution implies that the yield displacement of an element is independent of the yield strength. This behavior has been found to be true for several steel and reinforced concrete systems [20-22] and stiffness-proportional strength distribution has been recommended for asymmetric-plan systems in several recent investigations [22,23]. Note that the strength distribution used in this investigation does not require explicit calculation of the yield displacement.

\section{Symmetric-plan system}

The corresponding symmetric-plan system was defined as a system with coincidental CM, CR, and CSD, but with relative location and stiffness of all resisting elements as well as location and damping coefficient of all supplemental dampers identical to those in the asymmetricplan system. In other words, the corresponding symmetric-plan system is identical to the asymmetric-plan system but with rotational degree of freedom restrained. Therefore, the symmetric plan system has only one degree-of-freedom: translation in the direction of applied force (or ground motion). For the one-story system selected in this investigation, the symmetric-plan system can be replaced by a single-degree-of-freedom (SDOF) system with mass, stiffness, and damping coefficient equal to the total mass, total stiffness of all lateral load resisting elements, and total damping coefficient of all dampers, respectively, in the symmetric-plan system.

\section{Ground motions}

The sets of 20 ground motion records were assembled for Los Angeles, Seattle, and Boston representing probabilities of exceedance of $2 \%, 10 \%$, and $50 \%$ in 50 years (return periods of 2475,475 , and 72 years, respectively) [16]. The $10 \%$ probability of exceedance in 50 years set of records developed for Los Angeles are used in this investigation.

\section{Response statistics}

The dynamic response of each system to each of 20 ground motions is determined by response history analysis [11]. Presented in this paper are median values $\hat{x}$, defined as the geometric mean, of $n(=20)$ observed values of $x_{i}$ of the peak value of the structural response [24]:

$$
\hat{x}=\exp \left(\sum_{i=1}^{n} \ln x_{i} / n\right)
$$



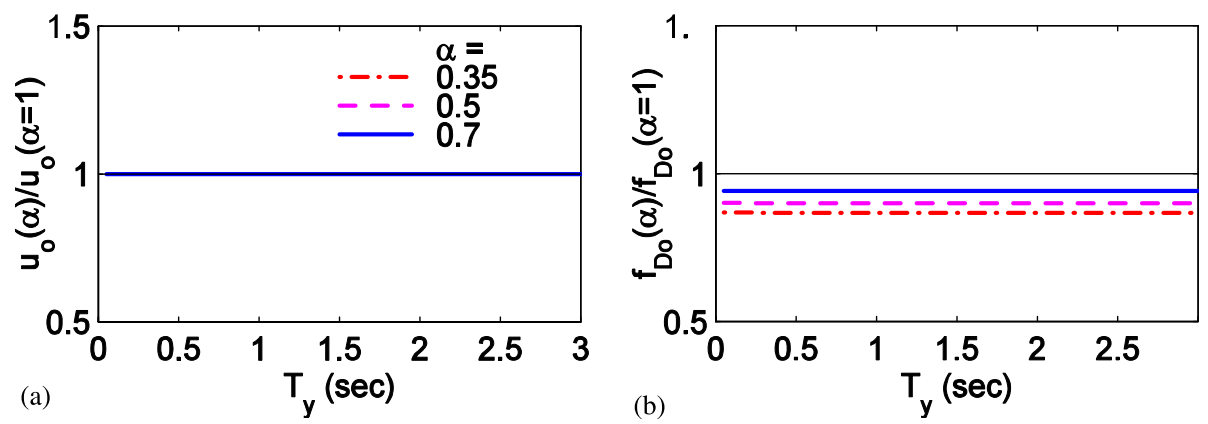

Figure 3. Ratio of steady-state response of SDOF systems with non-linear and linear damper subjected to harmonic loading: (a) deformation; and (b) damper force.

\section{RESPONSE OF SINGLE-DEGREE-OF-FREEDOM SYSTEMS}

Prior to investigating the seismic response of asymmetric-plan systems with supplemental dampers, it is useful to review the response behaviour of symmetric-plan, or single-degree-offreedom (SDOF) systems, with linear and non-linear fluid viscous dampers. For this purpose, the responses of SDOF systems to two types of loading-harmonic and earthquake-are examined in this section. The response for harmonic loading is presented for linear systems, whereas the response of linear and non-linear systems are presented for the earthquake loading. For non-linear systems, the strength is defined by reducing the strength required for the system to remain elastic by a reduction factor $R=4$. Furthermore, the damping coefficient, $C$, for nonlinear systems is kept the same as that for the corresponding elastic system, implying that the non-linear system in the elastic range of behaviour would have the same equivalent damping ratio as the corresponding linear system. The response quantities examined in this section are the ratios of peak deformations, $u_{\mathrm{o}}() / u_{\mathrm{o}}(=1)$, and peak damper force, $f_{\mathrm{Do}}() / f_{\mathrm{Do}}(=1)$, in a SDOF system with non-linear and linear damper, both systems with the same equivalent damping ratio, $s d=20 \%$.

\section{Harmonic loading}

Presented in Figure 3 are the results for steady-state response of linear SDOF systems subjected to harmonic loading with excitation frequency equal to natural vibration frequency of the system, i.e. $\omega=\omega_{\mathrm{n}}$ (or $T=T_{\mathrm{n}}$ ). The presented results show that the ratio $u_{\mathrm{o}}() / u_{\mathrm{o}}(=1)=1$ for all period values and is independent of the damper non-linearity, , as curves for all values are identical (Figure 3(a)), a result that is expected because the definition of equivalent damping ratio for SDOF systems with non-linear dampers is based on harmonic loading. The ratio $f_{\mathrm{Do}}() / f_{\mathrm{Do}}(=1)$ is also independent of the period but depends on the value (Figure 3(b)). Since the ratio $V / \dot{u}_{\mathrm{o}}$ in Equation (9) is equal to one for steady-state response of linear SDOF systems subjected to harmonic loading with $\omega=\omega_{\mathrm{n}}$, the ratio $f_{\mathrm{Do}}() / f_{\mathrm{Do}}(=1)$ $=/ \beta$, which depends only on the damper non-linearity, , and is independent of the system period, as confirmed by the results of Figure $3(\mathrm{~b})$. The ratio $f_{\mathrm{Do}}() / f_{\mathrm{Do}}(=1)$ is always less than one for non-linear dampers, i.e. $\alpha<1$. 

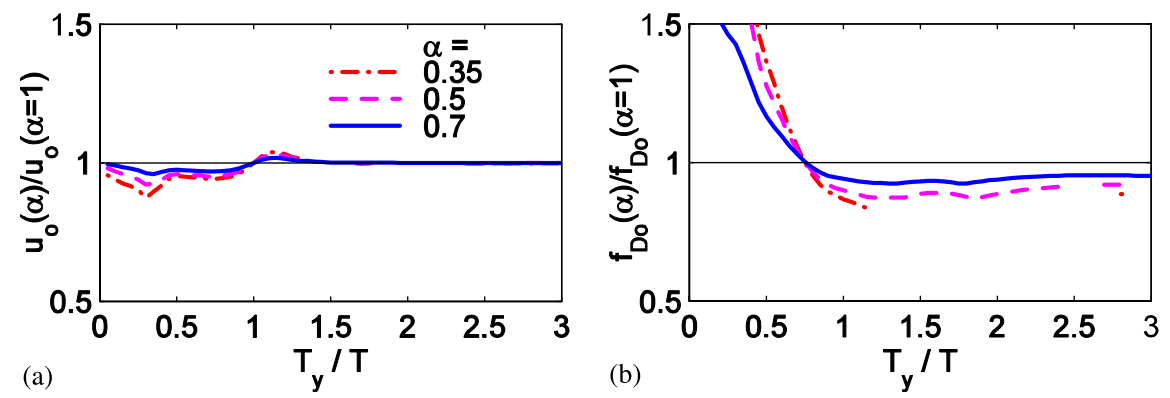

Figure 4. Ratio of steady-state response of SDOF systems with non-linear and linear damper subjected to a fixed harmonic loading: (a) deformation; and (b) damper force.

The results presented in Figure 3 are for SDOF systems subjected to excitation frequency equal to the natural vibration frequency of the system, i.e. $\omega=\omega_{\mathrm{n}}$. It is also useful to examine the responses over a range of natural frequencies (or periods) of the SDOF system but with fixed excitation frequency. For this purpose, responses were computed for SDOF systems with vibration periods in the range of 0.05 to $3 \mathrm{~s}$ subjected to harmonic loading with period of $1 \mathrm{~s}$. The results presented in Figure 4 show that $u_{0}() / u_{0}(=1)$ is less than one for $T_{y} / T<1$, becomes slightly more than one for $1<T_{y} / T<1.5$, and is essentially equal to one for $T_{y} / T>1.5$ (Figure 4(a)). For $T_{y} / T=1$, the ratio $u_{\mathrm{o}}() / u_{\mathrm{o}}(=1)$ is exactly equal to one, which is consistent with the results presented in Figure $3(\mathrm{a})$. The ratio $f_{\mathrm{Do}}() / f_{\text {Do }}(=1)$ is much larger than one for systems with $T_{y} / T$ values less than a cutoff value and smaller than one for $T_{y} / T$ values more than a cutoff value (Figure 4(b)); the cutoff value of $T_{y} / T$ is slightly less than one. Furthermore, these effects become larger with increasing damper non-linearity.

The results presented in Figure 4 imply that damper non-linearity reduces deformations but increases damper force for systems with periods shorter than the excitation frequency. For longer period systems, the deformations are essentially unaffected and the damper force is reduced due to damper non-linearity.

\section{Earthquake loading}

Figure 5 presents median values of results for linear elastic systems subjected to the selected ensemble of ground motions. These results show that the ratio $u_{0}() / u_{0}(=1)$ is slightly less than one for system periods less than about $1.5 \mathrm{~s}$ (Figure 5(a)) indicating that the peak deformation of the elastic SDOF system with non-linear damper is slightly less than that of the SDOF system with linear damper, both with the same amount of equivalent damping. For system periods longer than about $1.5 \mathrm{~s}$, the ratio $u_{\mathrm{o}}() / u_{\mathrm{o}}(=1)$ approaches one indicating essentially no reduction in deformation due to damper non-linearity. The ratio $f_{\mathrm{Do}}() / f_{\mathrm{Do}}(=1)$ may become larger than one for systems with very short periods $\left(T_{y}<0.2 \mathrm{~s}\right)$ (Figure 5(b)) indicating an increase in damper force due to damper non-linearity. For longer period systems, however, the ratio $f_{\mathrm{Do}}() / f_{\mathrm{Do}}(=1)$ is less than one implying reduction in damper force due to damper non-linearity. These effects increase with increasing damper non-linearity (or decreasing value of ).

Some of the trends apparent from the results presented in Figure 5 cannot be explained based on the response of the SDOF system subjected to harmonic loading with $\omega=\omega_{\mathrm{n}}$, a loading 

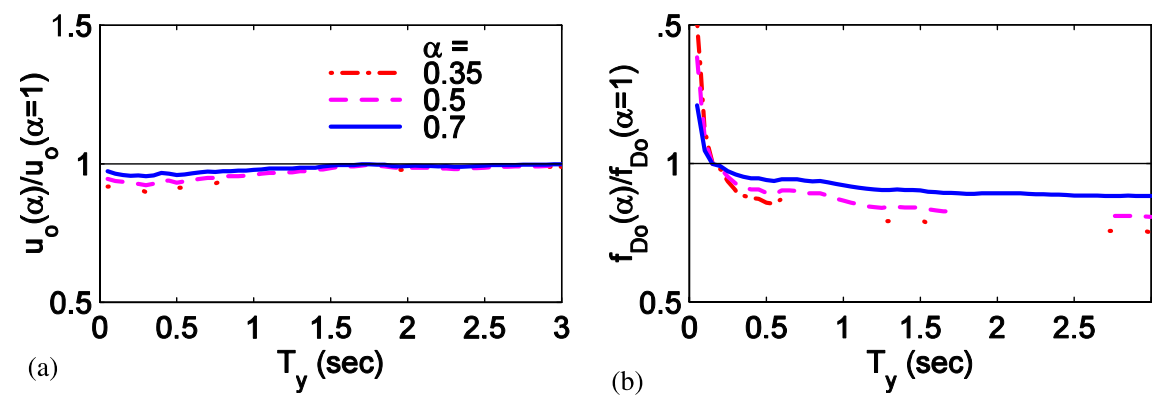

Figure 5. Median ratio of response of linear SDOF systems with non-linear and linear damper subjected to an ensemble of ground motions: (a) deformation; and (b) damper force.
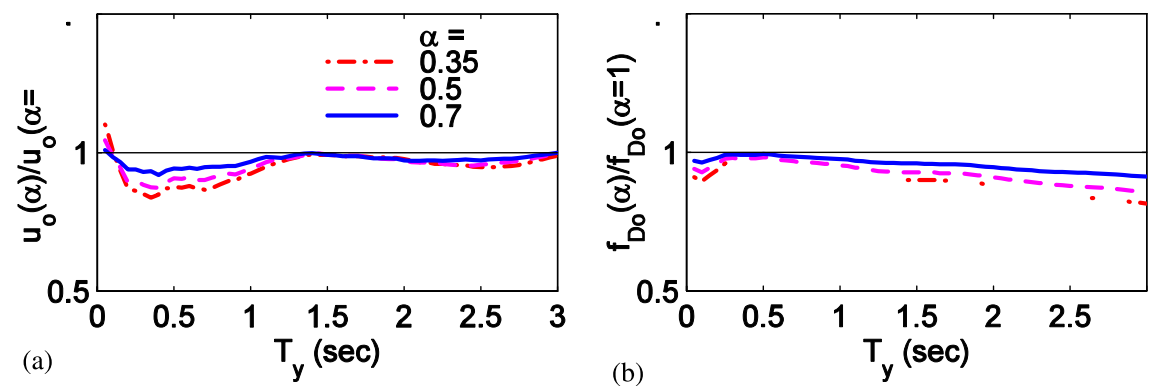

Figure 6. Median ratio of response of non-linear SDOF systems with non-linear and linear damper subjected to an ensemble of ground motions: (a) deformation; and (b) damper force.

that is widely used for simplified design of systems with non-linear dampers. For example, the deformations due to earthquake loading are reduced and damper force increased by damper non-linearity for short-period systems (Figures 5(a) and (b)). The results presented in Figure 3 for the traditionally used harmonic loading, on the other hand, indicated that deformations are unaffected and the damper forces are reduced by damper non-linearity for such systems. Such is the case because both loading characteristics (loading frequency) and the system vibration period are varied together by enforcing $\omega=\omega_{\mathrm{n}}$ for results in Figure 3 . For the earthquake loading, however, the loading characteristics remain unchanged (same earthquake ground motion) while the vibration period is varied. Therefore, the earthquake and harmonic loadings lead to different trends for short-period systems.

An improved correlation is obtained by examining the results for a fixed harmonic loading and varying the vibration period of the system. The results presented in Figure 4 indicate that deformation reduces and damper force increases with damper non-linearity for short-period systems, trends that are consistent with those in Figure 5 for earthquake loading.

Figure 6 presents median values of results for non-linear systems subjected to the selected ensemble of ground motions. These results show that the ratio $u_{0}() / u_{0}(=1)$ is less than one for non-linear systems with non-linear dampers (Figure 6(a)), with the exception at very short periods where the ratio may become slightly larger than one. The reduction in $u_{\mathrm{o}}() / u_{\mathrm{o}}(=1)$ 

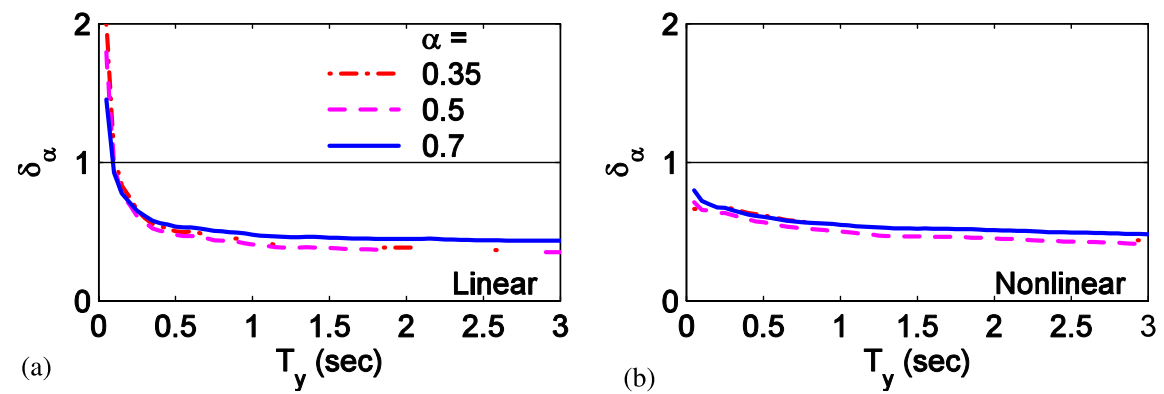

Figure 7. Median values of ratio for SDOF systems subjected to an ensemble of ground motions: (a) linear elastic systems; and (b) non-linear systems.

for non-linear systems appears to be slightly larger than that for linear systems. The increase in the ratio $f_{\mathrm{Do}}() / f_{\mathrm{Do}}(=1)$ noted for linear systems for very-short-period systems (Figure 4(b)) does not occur for non-linear systems (Figure 6(b)). Furthermore, the reduction in $f_{\mathrm{Do}}() / f_{\mathrm{Do}}(=1)$ for longer period non-linear systems is smaller than for the linear systems.

The above-noted difference in trends in the damper forces between linear and non-linear systems may be explained by careful examination of the following relationship between the damper force in SDOF systems with non-linear and linear dampers having the same amount of equivalent damping ratio:

$$
\frac{f_{\mathrm{Do}}()}{f_{\mathrm{Do}}(=1)}=\frac{1}{\left(\omega_{\mathrm{n}} u_{\mathrm{oe}, \alpha}\right)^{1-}} \frac{\left(\dot{u}_{\mathrm{o}, \alpha}\right)}{\dot{u}_{\mathrm{o}, \alpha=1}}
$$

in which $u_{\mathrm{oe}, \alpha}$ is the peak deformation of the linear SDOF system with non-linear damper, and $\dot{u}_{\mathrm{o}, \alpha}$ and $\dot{u}_{\mathrm{o}, \alpha=1}$ are the peak velocities of the SDOF system (linear or non-linear) with nonlinear damper (velocity exponent ) and linear damper (velocity exponent $=1$ ), respectively.

Equation (10) shows that the ratio $f_{\mathrm{Do}}() / f_{\mathrm{Do}}(=1)$ for earthquake loading depends on the ratio $=\left(\omega_{\mathrm{n}} u_{\mathrm{oe}, \alpha}\right)^{1-}\left(\dot{u}_{\mathrm{o}, \alpha}\right) / \dot{u}_{\mathrm{o}, \alpha=1}$ in addition to the parameter defined by Equation (4). The simplification achieved in Equation $(9)$, where $f_{\mathrm{Do}}() / f_{\mathrm{Do}}(=1)$ depends on $\left(V / \dot{u}_{\mathrm{o}}\right)^{1-}$ is not possible for earthquake loading because the peak velocity of the SDOF system with non-linear damper may not be equal to that of the corresponding SDOF system with linear damper, as apparent from the deformation ratios $u_{0}() / u_{0}(=1)$ presented in Figures 5 and 6 .

The median values of the ratio for the selected ensemble of ground motions are presented in Figure 7 for linear and non-linear systems. These results show that values of exceed one for very-short-period linear elastic systems (Figure 7(a)) implying that the damper force in very-short-period elastic systems may be larger in the non-linear damper compared to the linear damper (Figure 5(b)). For very-short-period non-linear systems, however, values of do not exceed one (Figure 7(b)) indicating that the damper force in non-linear systems would not exceed that in the linear damper (Figure 6(b)). Smaller reductions in $f_{\text {Do }}() / f_{\text {Do }}(=1)$ occur for longer period non-linear systems due to damper non-linearity compared to the linear systems (see Figures 5(b) and 6(b)) because values of are larger for the former compared to the latter (see Figures $7(\mathrm{a})$ and (b)). 


\section{RESPONSE OF ASYMMETRIC SYSTEMS}

\section{System parameters}

The linear response of one-story, asymmetric-plan systems without supplemental damping depends on (1) transverse vibration period, $T_{y}=2 \pi / \omega_{y}$ ( $\omega_{y}=$ vibration frequency), of the corresponding symmetric-plan system in the $y$-direction; (2) normalized stiffness eccentricity, $e=e / a(a=$ plan dimension perpendicular to the direction of ground motion); (3) ratio of the torsional and transverse frequencies, ; (4) aspect ratio of the deck, $a / d$; and (5) mass and stiffness proportional damping constants, $a_{0}$ and $a_{1}$, which in turn depend on the natural damping ratios in the two vibration modes of the system. The additional parameters needed to include supplemental damping are: (1) supplemental damping ratio, sd; (2) normalized supplemental damping eccentricity, $e_{\mathrm{sd}}=e_{\mathrm{sd}} / a$; and (3) damper velocity exponent, . A detailed description of various parameters is available elsewhere [14, 19].

Responses are presented for the following values of system parameters: $T_{y}$ in the range of 0.05 to $3 \mathrm{~s} ; \quad=1 ; e=0.2$; aspect ratio $=2$; and $=5 \%$ in all modes of the corresponding linear elastic symmetric-plan system. The parameters for the supplemental damping system were selected as: ${ }_{\mathrm{sd}}=20 \% ; e_{\mathrm{sd}}=-0.2$; and $=1,0.7,0.5$, and 0.35 . For non-linear systems, the reduction factor has been selected to be the same in both directions, i.e. $R_{x}=R_{y}=4$.

\section{Response quantities considered}

The following six response quantities are considered in this investigation: stiff- and flexibleedge deformations; base shear and base torque; and total damping force and damping torque at the base of the system. The base shear and base torque considered in this investigation include force contributions from lateral load resisting elements as well as supplemental dampers. Among these responses, the edge deformations have generally received the most scrutiny [1]. However, it is useful to examine the various force quantities because it would enable verifying the assertion that damper non-linearity limits the force in the damper.

\section{Effects of damper non-linearity}

In order to evaluate the effects of damper non-linearity on the seismic response of asymmetric plan systems, ratios of the selected responses in asymmetric-plan systems with non-linear and linear dampers are computed for each ground motion and their median values determined. These ratios facilitate whether the response is amplified (increased) or de-amplified (reduced) due to damper non-linearity. In order to establish a baseline for comparison, the results are presented first for systems with lateral load-resisting elements responding in the linear elastic range (Figure 8); these systems are denoted as 'linear' systems. Subsequently, results are presented for systems with lateral load-resisting elements deformed beyond the elastic limit (Figure 9); these systems are denoted as 'non-linear' systems. Note that the distinction between 'linear' and 'non-linear' systems is only due to the behaviour of the lateral load-resisting elements; both these systems may have non-linearity associated with dampers. The response ratios are computed such that both asymmetric systems, with non-linear and linear dampers, are either 'linear' or 'non-linear'.

The results for linear systems show that the damper non-linearity reduces stiff- and flexibleedge deformations over a wide range of period values (Figures $8(a)$ and (b)). Furthermore, 

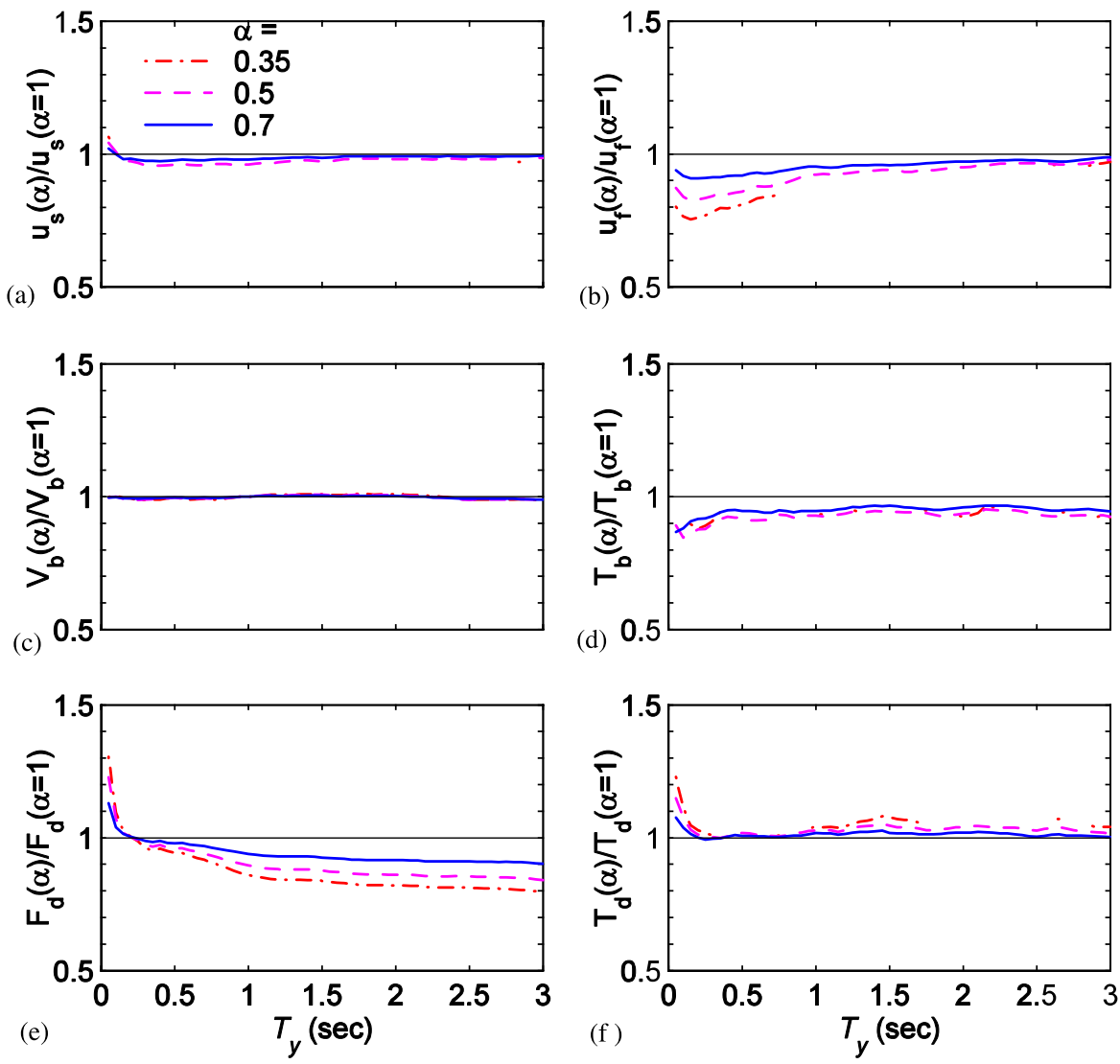

Figure 8. Median values of ratios of seismic responses of asymmetric-plan systems with non-linear $(=0.7,0.5$, and 0.35$)$ and linear $(=1)$ fluid viscous dampers: linear systems.

higher damper non-linearity leads to larger reduction in the deformations. For the flexibleedge, reductions up to $25 \%$ may be achieved for short-period $\left(T_{y}<0.5 \mathrm{~s}\right)$ systems; reductions are much smaller (less than 10\%) for longer period systems (Figure 5(b)). The reductions for the stiff-edge deformations are minimal: the reductions are, in general, about $5 \%$ (Figure $8(\mathrm{a})$ ) for the lowest value of $=0.35$ considered in this investigation. For very-short-period systems, deformation of the stiff-edge may even increase slightly (Figure 8(a)). The reduction in deformation due to damper non-linearity is larger for the flexible-edge and smaller for the stiff-edge compared to that found previously for SDOF systems (compare Figures 8(a) and (b) with Figure 4(a)).

The damper non-linearity has little influence on the base shear as apparent from the ratio being nearly equal to one over the entire period range (Figure 8(c)). The base shear is insensitive to the damper non-linearity because deformation of the structural element on the stiff-edge side, which contributed the most to the base shear, is affected very little by the damper nonlinearity (Figure 8(a)). However, the base torque which occurs due to plan asymmetry, is reduced with the largest reduction of about $15 \%$ for $=0.35$ and systems with $T_{y}<0.5 \mathrm{~s}$ 

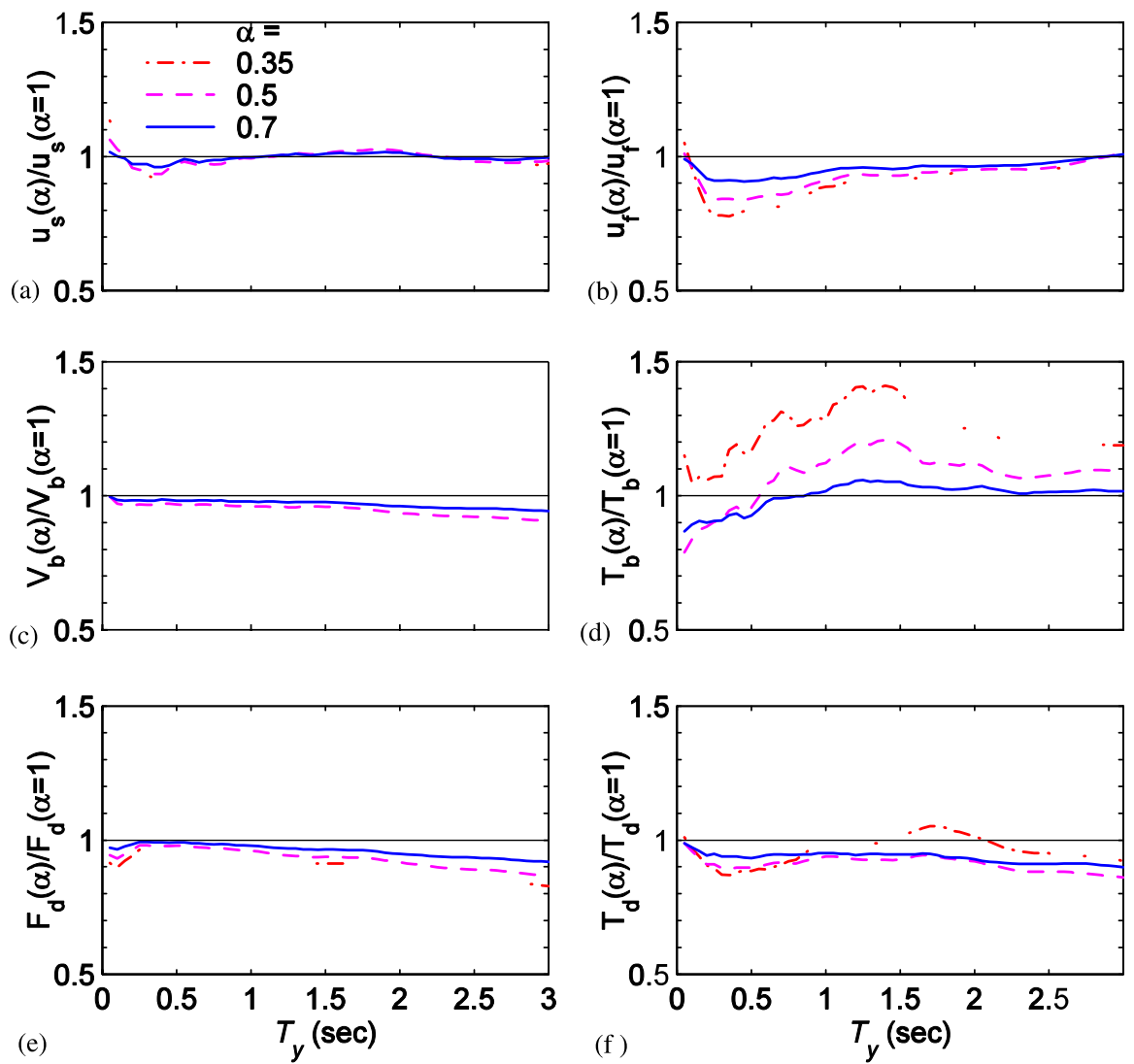

Figure 9. Median values of ratio of seismic response of asymmetric systems with non-linear $(=0.7$, 0.5 , and 0.35$)$ and linear $(=1)$ fluid viscous dampers: non-linear systems.

(Figure $8(\mathrm{~d})$ ). Such is the case because the flexible-edge element contributes significantly to the base torque about the CR due to the larger lever arm (see Figure 2(a)) and deformation of this element (and hence its contribution to the total torque) is significantly reduced by the damper non-linearity (Figure $8(\mathrm{~b}))$. For longer period systems $\left(T_{y}>2 \mathrm{~s}\right)$ reduction in the base torque is minimal.

The total damper force reduces, with a few exceptions, with increasing damper non-linearity, i.e. a reducing value of (Figure 8(e)). This observation is consistent with the earlier conclusions that the damper non-linearity reduces damper force (Figure 5(b)) reached based on responses of SDOF systems subjected to earthquake loading. The reduction is about $20 \%$ for $=0.35$ over the wide range of period values considered (Figure 8(e)). However, the exception occurs for very-short-period systems: the total damping force increases, instead of reducing, due to damper non-linearity (Figure $8(\mathrm{e})$ ). This increase may be as large as $30 \%$ (Figure 8(e)).

Owing to asymmetry in the system plan and the damper distribution, the damper forces are not symmetrically distributed about the geometric center of the system resulting in a damping 
torque. Note that the damping torque will not occur in symmetric systems with symmetric damping distribution. The presented results show that the total damping torque at the base increases slightly with increasing damper non-linearity (Figure 8(f)); the increase is less than $10 \%$ for $=0.35$ over a wide range of period values. For very-short-period systems, however, the increase in total damping torque may exceed $20 \%$.

Most previous investigations on seismic response of linear SDOF systems [6-10], as well as a recent investigation on seismic response of linear asymmetric systems [12], reached the conclusion that damper non-linearity reduces damper force significantly. Based on the results presented so far in this paper, however, it must be emphasized that this conclusion is strictly valid for systems with periods longer than about $0.2 \mathrm{~s}$. For systems with periods shorter than $0.2 \mathrm{~s}$, the damper force may increase due to damper non-linearity. Therefore, non-linear dampers for very-short-period systems should be used cautiously.

The results for the non-linear systems show that the damper non-linearity reduces edge deformations (Figures 9(a) and (b)). Reductions up to $25 \%$ in the flexible-edge deformation may be achieved for short-period systems, whereas the reductions in the stiff-edge deformations are generally less than 5\%. For very-short-period systems, the damper non-linearity may increase the stiff-edge deformations. These trends for non-linear systems are generally similar to those observed previously for linear systems.

However, the trends for base shear and base torque may differ between linear and nonlinear systems. While the base shear was unaffected by the damper non-linearity for the linear system over the entire period range (Figure 8(c)), it may reduce slightly for long period $\left(T_{y}>2 \mathrm{~s}\right)$ non-linear systems with the reduction between $5 \%$ and $10 \%$ (Figure $9(\mathrm{c})$ ). While the damper non-linearity reduces the base torque for linear systems (Figure 8(d)), it increases the base torque for non-linear systems with the increase of $20 \%$ or more over a wide range of periods for $=0.35$ (Figure $9(\mathrm{~d})$ ).

The damper non-linearity reduces the total damper force (Figure 9(e)). However, the reduction in damper force is slightly smaller for non-linear systems compared to the linear systems (compare Figures 9(e) and 8(e)). Furthermore, increases in damper force in veryshort-period systems noted for linear systems (Figure $8(\mathrm{e})$ ) do not occur for non-linear systems (Figure 9(e)). These trends are consistent with the trends noted previously for SDOF systems (Figures 5(b) and 6(b)).

The trends for the total damper torque in non-linear systems are not consistent over the period range considered in this investigation. While damper non-linearity reduces damper torque for short-period systems, it may reduce or increase the damper torque for longer period systems (Figure 9(e)). The values of reduction may be up to $15 \%$ and increase up to $5 \%$.

The results presented so far indicate that the damper non-linearity may be used to achieve reduction in the flexible-edge deformation of the order of $25 \%$ for short-period linear and non-linear systems $\left(T_{y}<0.5 \mathrm{~s}\right)$. Furthermore, the damper non-linearity may reduce the total damping force between $15 \%$ and $20 \%$. However, such reduction occurs for systems with periods longer than about $0.2 \mathrm{~s}$. For systems with periods shorter than $0.2 \mathrm{~s}$, damper nonlinearity may lead to an increase in damper force indicating that dampers for such systems should be used cautiously.

The response of the 'non-linear' system presented in this section is strictly affected by two factors: (1) the damper non-linearity, and (2) a different effective (or apparent) damping ratio in the non-linear asymmetric system compared to that for the corresponding elastic SDOF 

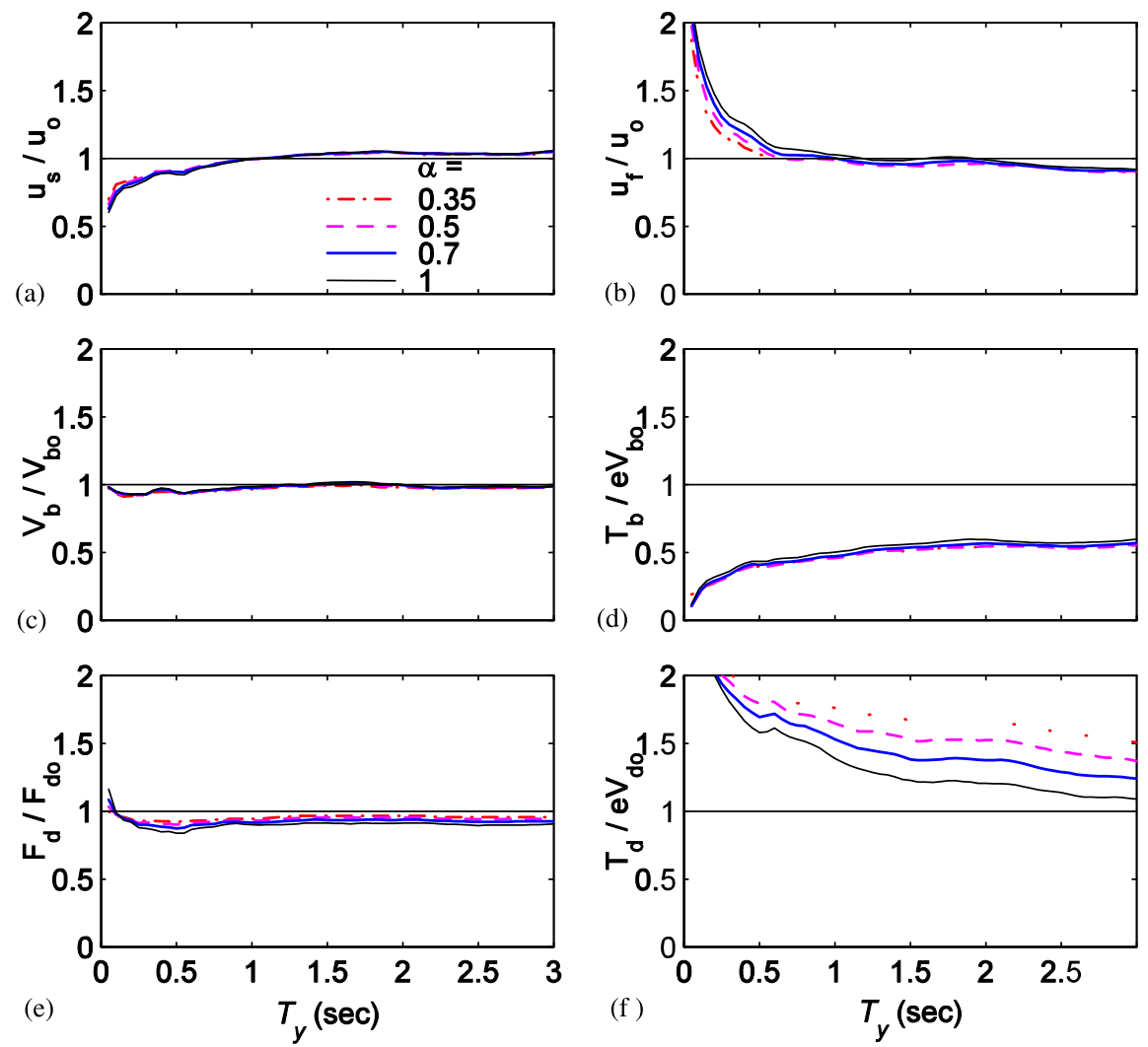

Figure 10. Effects of plan asymmetry in seismic response of asymmetric-plan systems with non-linear $(=0.7,0.5$, and 0.35$)$ and linear $(=1)$ fluid viscous dampers: linear systems.

system. While these two effects cannot be completely isolated for 'non-linear' systems because Equations (5) and (6) are strictly applicable for linear systems, the second effect is minimized by investigating the response ratio of asymmetric systems with non-linear and linear damping both responding in the 'non-linear' range; the two systems are likely to be affected in a similar way by the second effect.

\section{Influence of damper non-linearity on effects of plan asymmetry}

Influence of damper non-linearity on the effects of plan asymmetry are evaluated by examining the median of ratios of the response of the asymmetric-plan system and its corresponding symmetric-plan system for a range of values; the corresponding symmetric-plan system will have the same damping ratio, sd, and velocity exponent, as the asymmetric-plan system. The median values of the ratio for six response quantities are presented in Figures 10 and 11 for linear and non-linear systems, respectively. Summarized in this section first are the effects of plan asymmetry in the system without supplemental damping. Subsequently, the results are compared for systems with linear and non-linear supplemental dampers to examine how the effects of plan asymmetry are modified by the damper non-linearity. 

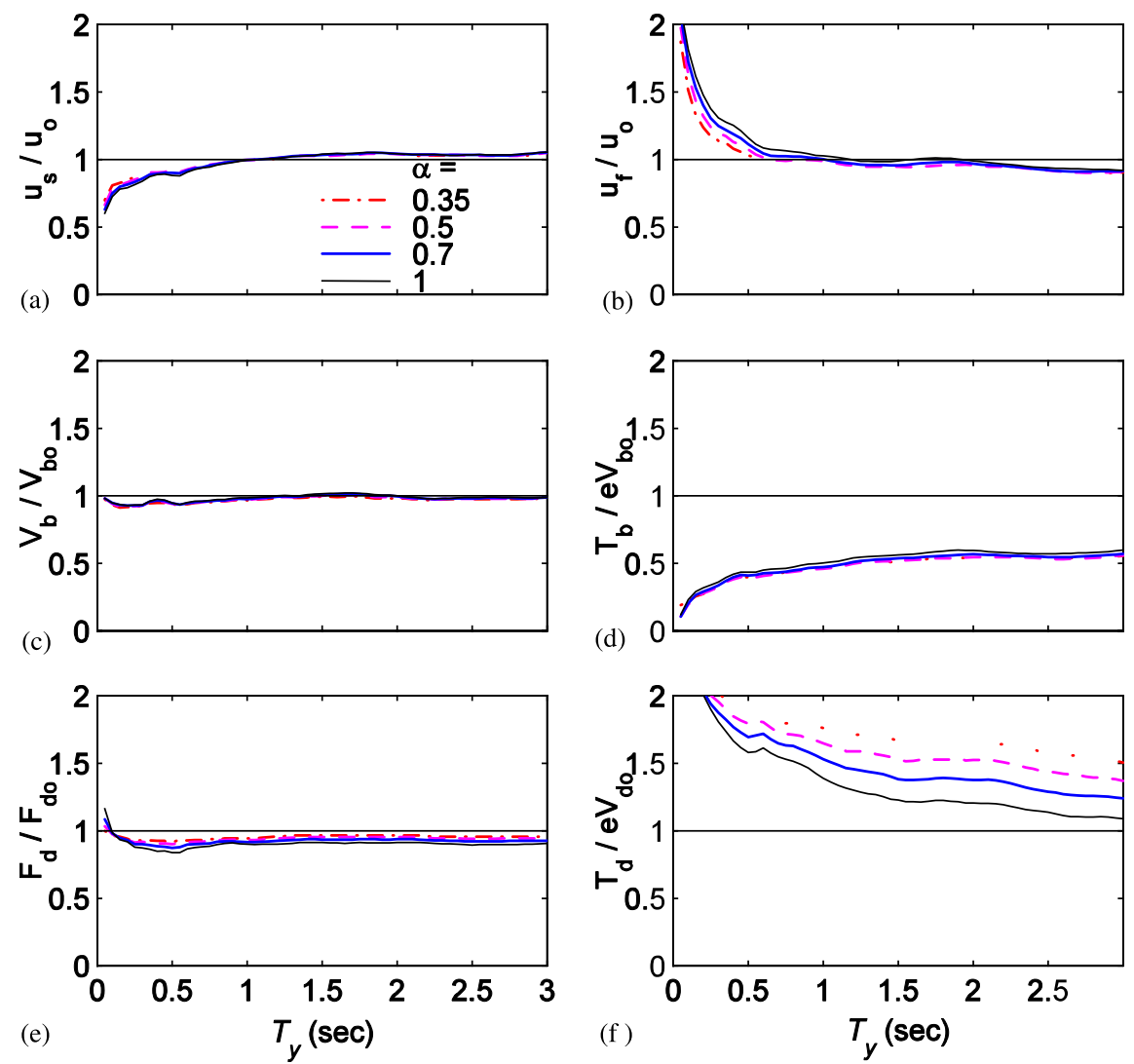

Figure 11. Effects of plan asymmetry in seismic response of asymmetric-plan systems with non-linear $(=0.7,0.5$, and 0.35$)$ and linear $(=1)$ fluid viscous dampers: non-linear systems.

The effects of plan asymmetry in linear systems without supplemental damping include an increase in deformation of the flexible-edge, decrease in the deformation of the stiff-edge, and reduction in the base shear compared to those in the corresponding symmetric plan system $[25,26]$. Furthermore, plan asymmetry induces base torque in asymmetric systems, which does not occur in the corresponding symmetric-plan system. These effects are expected to become less prominent in systems with supplemental damping [1].

The presented results for linear systems with supplemental damping show that deformation of the stiff-edge is smaller, and flexible-edge larger, in asymmetric-plan systems compared to the corresponding symmetric-plan system (Figures 10(a) and (b)). This trend is consistent with the effects of plan asymmetry summarized previously. However, these effects are prominent only for short-period systems: $T_{y}$ up to $0.5 \mathrm{~s}$. For systems with period longer than $0.5 \mathrm{~s}$, the effects of plan asymmetry on the edge deformations are minimal. The damper nonlinearity appears to slightly reduce the effects of plan asymmetry; the decrease in the stiff-edge deformation (Figure 10(a)) and increase in the flexible-edge deformation (Figure 10(b)) are slightly smaller for lower values of . 
The effects of plan asymmetry, which reduces the stiff-edge deformation and increases the flexible-edge deformation of the linear systems, are almost non-existent for non-linear systems (Figures 11(a) and (b)) as apparent from the ratios being close to one over the entire period range. This occurs due to doubling of effects of supplemental damping [1] and system nonlinearity [25] both of which have been shown to reduce the effects of plan asymmetry. The damper non-linearity influences the deformations of non-linear systems in a similar way as for the linear systems, i.e. smaller flexible-edge deformation and larger stiff-edge deformations with increasing damper non-linearity. However, these effects are not noticeable for non-linear systems because of much smaller effects of plan asymmetry.

The results presented so far for the edge deformations indicate that the damper non-linearity reduces the adverse effects of plan asymmetry, i.e. increase in the flexible-edge deformation and decrease in the stiff-edge deformation of the asymmetric-plan systems compared to the same edges of the corresponding symmetric-plan systems. Furthermore, using a combination of non-linear supplemental damping and system non-linearity can eliminate the adverse effects of plan asymmetry. In particular, essentially identical values of the edge deformations may be obtained in non-linear asymmetric- and symmetric-plan systems.

The effects of damper non-linearity on base shear are negligibly small for linear as well as non-linear systems (Figures 10(c) and 11(c)). While the base torque for linear systems remains essentially unaffected by the damper non-linearity (Figure 10(d)), it is slightly increased for the non-linear systems (Figure 11(d)).

The plan asymmetry slightly reduces the total damping force in linear systems as apparent from the ratio being somewhat smaller than one for most period values (Figure 10(e)). The damper non-linearity influences these effects minimally as apparent from the curves for all values being very close. For non-linear systems, the ratio is essentially one for all values of

over the entire period range (Figure 11(e)) indicating that effects of plan asymmetry for such systems are minimal and they are not influenced by the damper non-linearity.

The combination of plan asymmetry and asymmetric damper distribution gives rise to the total damping torque at the base. For linear systems, the damping torque tends to increase with increasing damper non-linearity, i.e. decreasing values of (Figure 10(f)). A similar but much smaller increase occurs for the non-linear systems (Figure 11(f)). While the median values of the ratio are in general more than one for linear systems (Figure 10(f)), they are always less than one for non-linear systems (Figure 11(f)) indicating that the damping torque generated in non-linear systems are in general smaller than those in linear systems.

\section{DESIGN IMPLICATIONS}

The results presented in this paper on seismic response of symmetric and asymmetric systems with linear and non-linear dampers lead to two important design implications: (1) the effectiveness of the non-linear fluid viscous dampers in reducing damper forces as well as system deformations, and (2) complexity of the analysis of systems with non-linear systems. The following is a brief discussion of the design implications of the results presented in this paper.

The non-linear fluid viscous dampers are attractive for seismic response control of systems because they are expected to lead to better performance, e.g. smaller deformations, while 
limiting the damper force. The results presented in this investigation show that both these expectations are not necessarily met simultaneously for a selected system. For example, noticeable reduction in damper force occurs for longer period systems (see Figures 5(b) and 6(b) for SDOF systems, and Figures 8(e) and 9(e) for asymmetric plan systems) due to damper non-linearity. For very-short-period systems, however, damper non-linearity may lead to an increase in the damper force. The deformations, on the other hand, are reduced by damper non-linearity for short-period systems (see Figures 5(a) and 6(a) for SDOF systems, and Figures 8(b) and 9(b) for asymmetric plan systems). Therefore, the designer can obtain deformation reduction but at the cost of higher damper force for short-period systems and damper force reduction but without deformation reduction for longer period systems by using a non-linear damper instead of a linear damper.

There are several complexities associated with the analysis of systems with non-linear dampers. First, the damping coefficient associated with a specified (or desired) damping ratio depends on the peak deformation of the system (see Equation (6)) which itself depends on the damping ratio. Therefore, an iterative procedure is required for specifying the damping coefficient. No such iterations are required to define the damping coefficient of a linear damper. Second, for a selected damping coefficient, the equivalent damping ratio depends on the peak system deformation (see Equation (5)). This indicates that a system designed for a selected damping ratio during a design earthquake would provide different damping for earthquakes smaller or larger than the design earthquake: the damping ratio would be higher for smaller earthquakes and lower for larger earthquakes compared to the designed damping ratio. Linear fluid viscous dampers, on the other hand, provide the same damping ratio for all earthquake excitation. Finally, the damper non-linearity requires that equations of motion be solved directly in the time-domain. Although simplified procedures have been developed for the analysis of systems with non-linear dampers [13,15], the errors associated with approximations in the simplified analysis, which may be as large as $20 \%$ [13], would negate the advantage gained by the non-linear dampers in terms of response reduction. For most systems with linear dampers, the response can easily be computed using standard and well-understood response spectrum analysis methods. Therefore, the advantages of using non-linear dampers, i.e. reduction in flexible-edge deformation and damper force by about $20 \%$, must be weighed against the disadvantage associated with complexities of the analysis procedure or the error in the simplified procedure that may also approach $20 \%$.

\section{CONCLUSIONS}

The investigation examined the influence of damper non-linearity on the seismic response of one-story, one-way asymmetric linear and non-linear systems. Reviewed first is the behaviour of SDOF systems with non-linear dampers. While some of the trends from SDOF systems confirm the well-known findings on the influence of damper non-linearity, others have not been adequately explained in the past. In particular, the system deformation reduces and damper force increases for short-period linear systems due to damper non-linearity when subjected to earthquake loading. These trends cannot be explained based on the traditionally used harmonic loading with forcing frequency equal to the natural vibration frequency of the system, which indicates no influence on the deformation and reduction in the damper force. In this investigation, the trends for the earthquake loading have been explained based on an alternative 
approach in which the response of systems over a range of vibration periods (or frequencies) are computed for a harmonic loading with fixed frequency. Furthermore, the increase in damper force due to damper non-linearity noted for short-period linear systems does not occur for non-linear systems. This is the case because the ratio $=\left(\omega_{\mathrm{n}} u_{\mathrm{oe}, \alpha}\right)^{1-}\left(\dot{u}_{\mathrm{o}, \alpha}\right) / \dot{u}_{\mathrm{o}, \alpha=1}$ that governs the amplification (or de-amplification) of the damper force (Equation (10)) is much smaller for non-linear systems compared to the linear systems.

Subsequently, the seismic responses of asymmetric-plan systems with linear and non-linear dampers are compared to evaluate the effects of damper non-linearity and its influence on the effects of plan asymmetry. This investigation has led to the following important conclusions:

1. The damper non-linearity leads to reductions (up to $25 \%$ ) in the flexible-edge deformations of short-period $\left(T_{y}<0.5 \mathrm{~s}\right)$ systems. For longer period systems, however, the reduction is much more modest (about $10 \%$ ). The effects of damper non-linearity on the stiff-edge deformations are minimal with a reduction of no more than $5 \%$. These effects are found to be similar for both linear and non-linear systems.

2. The effects of damper non-linearity on base shear and base torque are found to be slightly different for linear and non-linear systems. While the damper non-linearity does not influence the base shear in linear systems, it may reduce the base shear in non-linear systems slightly (between $5 \%$ and $10 \%$ ) for $T_{y}>2 \mathrm{~s}$. The damper non-linearity reduces base torque for linear systems with reductions of up to $20 \%$ for $T_{y}<0.5 \mathrm{~s}$, whereas it increases the base torque for non-linear systems by more than $20 \%$ over a wide range of period values.

3. The damper non-linearity in general reduces the total damping force by about $15 \%$ and increases the total damping torque by about $10 \%$. These trends are similar for both linear and non-linear systems.

4. The reduction in the damper force is restricted to systems with periods longer than $0.2 \mathrm{~s}$. For systems with periods shorter than $0.2 \mathrm{~s}$, however, the damper non-linearity may significantly increase the damping force; increases in excess of $25 \%$ were found to occur. Therefore, non-linear dampers for such systems should be used cautiously. These increases in damper force occurred only for short-period linear systems.

5. The damper non-linearity reduces the effects of plan asymmetry leading to a smaller increase in the flexible-edge deformation and a smaller decrease in the stiff-edge deformation of the asymmetric-plan systems compared to the same edges of the corresponding symmetric-plan systems. This trend was found to occur for both linear and non-linear systems.

6. The effects of plan asymmetry on base shear are not influenced by the damper nonlinearity for both linear and non-linear systems. However, the damper non-linearity reduces the base torques slightly for linear systems but increases it for non-linear systems.

7. The plan asymmetry reduces the total damping force and introduces damping torque. While the damper non-linearity has very little influence on the total damping force, it tends to increase the damping torque for both linear and non-linear systems.

8. A combination of damper non-linearity and system non-linearity may be used to nearly eliminate the adverse effects of plan asymmetry. In particular, essentially identical values of the flexible- and stiff-edge deformations may be obtained in asymmetric- and symmetric-plan systems. 
Finally, design implications of the results presented in this paper have been discussed. In particular, it is noted that both reduction in deformations and damper forces due to damper non-linearity may not be obtained simultaneously for a selected system; while deformation reduction occurs for short-period systems, damper force reduction occurs for longer period systems. Furthermore, the advantages of the reduction in response by damper non-linearity may be negated by disadvantages associated with complexities in the analytical procedure and errors in the simplified procedures.

\section{ACKNOWLEDGEMENTS}

This research investigation was funded by the National Science Foundation under Grant CMS-9812414. This financial support is gratefully acknowledged. Suggestions from anonymous reviewers helped in significantly improving this manuscript.

\section{REFERENCES}

1. Goel RK. Effects of supplemental viscous damping on seismic response of asymmetric-plan systems. Earthquake Engineering and Structural Dynamics 1998; 27:125-141.

2. Goel RK. Seismic behaviour of asymmetric buildings with supplemental damping. Earthquake Engineering and Structural Dynamics 2000; 29:461-480.

3. Goel RK, Booker CA. Effects of supplemental viscous damping on inelastic seismic response of asymmetric systems. Earthquake Engineering and Structural Dynamics 2001; 30:411-430.

4. Lin WH, Chopra AK. Improving the seismic response of asymmetric one-storey systems by supplemental viscous damping. Proceedings of the Seventh U.S. National Conference on Earthquake Engineering. Engineering Research Institute, Oakland, California, 2002.

5. Lin WH, Chopra AK. Understanding and predicting effects of supplemental viscous damping on seismic response of asymmetric one-storey systems. Earthquake Engineering and Structural Dynamics 2001; 30:1475-1494.

6. Hanson RD, Soong TT. Seismic Design with Supplemental Energy Dissipation Devices. Earthquake Engineering Research Institute, Oakland, CA, 2001.

7. Asher JW, Young RP, Ewing RD. Seismic isolation design of the San Bernardino county medical center replacement project. Journal of Structure and Design of Tall Buildings 1996; 5(4):265-279.

8. Pekcan G, Mander JB, Chen SS. Fundamental considerations for the design of non-linear viscous dampers. Earthquake Engineering and Structural Dynamics 1999; 28:1405-1425.

9. Seleemah AA, Constantinou MC. Investigation of Seismic Response of Buildings with Linear and Non-linear Fluid Viscous Dampers. Report No. NCEER-97-0004, National Center for Earthquake Engineering Research, Buffalo, New York, 1997.

10. Soong TT, Dargush GF. Passive Energy Dissipation Systems in Structural Engineering. Wiley: New York, 1997.

11. Chopra AK. Dynamics of Structures: Theory and Applications to Earthquake Engineering (2nd edn). PrenticeHall: Upper Saddle River, NJ, 2001.

12. Lin WH, Chopra AK. Asymmetric one-storey elastic systems with non-linear viscous and viscoelastic dampers: Earthquake response. Earthquake Engineering and Structural Dynamics 2003; 32:555-577. DOI: 10.1002/eqe.237.

13. Lin WH, Chopra AK. Asymmetric one-storey elastic systems with non-linear viscous and viscoelastic dampers: Simplified analysis and supplemental damping system design. Earthquake Engineering and Structural Dynamics 2003; 32:579-596. DOI: 10.1002/eqe.238.

14. Goel RK. Seismic Response Study of Asymmetric Systems with Linear and Nonlinear Fluid Viscous Dampers. Report No. CP/SEAM-2002/06, Department of Civil and Environmental Engineering, California Polytechnic State University, San Luis Obispo, CA, 2002.

15. ASCE. Prestandard and Commentary for the Seismic Rehabilitation of Buildings. Report No. FEMA-356, Federal Emergency Management Agency, Washington, D.C., 2000.

16. Somerville P, Smith N, Punyamurthula S, Sun J. Development of Ground Motion Time Histories for Phase 2 of the FEMA/SAC Steel Project. Report No. SAC Background Document Report No. SAC/BD-9/04, SAC Joint Venture, 555 University Ave., Sacramento, CA, 1997.

17. Soong TT, Constantinou MC. Passive and Active Structural Vibration Control in Civil Engineering. Springer: New York, 1994. 
18. Filiatrault A, Tremblay R, Wanitkorkul A. Performance evaluation of passive damping systems for the seismic retrofit of steel moment-resisting frames subjected to near-field ground motions. Earthquake Spectra 2001; 17(3):427-456.

19. Goel RK. Seismic response control of irregular structures using non-linear dampers. Proceedings of the 13th World Conference on Earthquake Engineering. Vancouver, B.C., Canada, 2004.

20. Priestley MJN. Performance based seismic design. Proceedings of the 12th World Conference on Earthquake Engineering. Auckland, New Zealand, 2000.

21. Aschheim MA. Seismic design based on yield displacement. Earthquake Spectra 2002; 18(4):581-600.

22. Paulay T. Seismic torsional effects on ductile structural wall systems. Journal of Earthquake Engineering 1997; 1(4):721-745.

23. Tso WK, Smith RSH. Re-evaluation of seismic torsional provisions. Earthquake Engineering and Structural Dynamics 1999; 28(8):899-917.

24. Benjamin JR, Cornell CA. Probability, Statistics, and Decision for Civil Engineers. McGraw-Hill: New York, p. $684,1970$.

25. Goel RK, Chopra AK. Inelastic Seismic Response of One-storey, Asymmetric-plan Systems. Report No. UCB/EERC-90/14, Earthquake Engineering Research Center, University of California, Berkeley, CA, 1990.

26. Hejal R, Chopra AK. Earthquake Response of Torsionally-coupled Buildings. Report No. UC/EERC-87/20, Earthquake Engineering Research Center, University of California, Berkeley, CA, 1987. 